\title{
Ergonomics principles to design clothing work for electrical workers in Colombia
}

\author{
Castillo, Juan ${ }^{\mathrm{a}, *}$, Cubillos, $\mathrm{A}^{\mathrm{b}}$ \\ ${ }^{a}$ Ergomotion laboratory, Health sciences and Medicine school. University of Rosario Bogota, Colombia \\ ${ }^{\mathrm{b}}$ Industrial Design school, Arts Faculty. National University of Colombia, Bogota Colombia
}

\begin{abstract}
The recent development of the Colombian legislation, have been identified the need to develop protective clothing to work according to specifications from the work done and in compliance with international standards. These involve the development and design of new strategies and measures for work clothing design. In this study we analyzes the activities of the workers in the electrical sector, the method analyzes the risks activity data in various activities, that activities include power generation plants, local facilities, industrial facilities and maintenance of urban and rural networks. The analyses method is focused on ergonomic approach, risk analysis is done, we evaluate the role of security expert and we use a design algorithm developed for this purpose. The result of this study is the identification of constraints and variables that contribute to the development of a model of analysis that leads to the development the work protective clothes.
\end{abstract}

Keywords: Activity analysis, Design, Ergonomics, Usability, User experience.

\section{Introduction}

Ergonomics is concerned with understanding and studying the work, that is to say, the activity made by the worker to achieve goals and objectives within the framework of a production system, the ergonomics tries to show as the activity is constructed and developed by the worker.

In this process of creation to experience and knowledge are developed, not only what they are required appropriately to reach the proposed objective, also to the components relative to the anticipation and the prevention are developed, since these allow to maintain the control of a situation and assure integrity to the worker.

In the study of a work activity in which the work clothes used constitutes an important and central component for controlling exposure to hazards specific to the work, the activity analysis takes into account the following concepts:
1. Comfort: is defined as a situation that is perceived as not unpleasant, where the human body should not make any effort to feel good.

2. Functionality has to do with the performance of the product, ie the ability to make proper use of this.

3 . Usability in the study means the product's suitability with respect to its use

Ergonomics applied to study the comfort of products, while associated with the functionality and the possible use of these specific work situations. In the evaluation of work clothes, the functionality of the components of work clothes should be linked to the complexity of the actions the worker must perform, this functionality should be associated with the comfort perceived by the worker in terms of thermal conditions, anthropometric and visual appearance. Finally the usability of the pieces will be appreciated by workers according to the possibilities these give the individual to meet the goals it has set for the development of assigned tasks, ergonomics in this perspective helps to identify how these elements should

*E-mail: juan.castillom@urosario.edu.co 
be integrated into the design of the work clothes of workers.

In this study the user experience is central to defining the final product characteristics. For this reason, the study aims to collect workers information from the user experience of work clothing, this in order to establish the usability of it and the criteria for improvement or design recommendations. On the other hand, the study is complemented by the analysis of the activities of workers in order to establish the relevance of work clothes that they wear, while evaluating the adaptation of these to the demands and requirements of the executed tasks.

It must be remembered that the design of protective clothing for workers in the electricity sector is defined by different standards; the main criterion used is the definition of risk categories, which are set according to the exposure of workers. These categories allow you to set the protection type and properties of the materials used. In NFPA 70E Standard for Electrical Safety Requirements for Employee Workplaces, was established the following types of risks:

1. Risk / Hazard Category 2: This level of protection could be obtained with more cotton underwear FR shirt and pants or FR shirt and pants of a single layer 4.6 - 9.8 oz fabric layer or as a lab coat.

2. Risk / Hazard Category 3: This level of protection could be obtained with cotton underwear plus FR shirt and pants, a shirt and pants or FR, more than a single layer coverall $9.8 \mathrm{oz}$ or $13 \mathrm{oz}$. FR layer, protection required safety glasses.

3. Risk / Hazard Category 4: This level of protection could be obtained with cotton underwear plus FR shirt and FR pants plus or $31^{2}$ $\mathrm{cal} / \mathrm{cm}, 40 \mathrm{cal} / \mathrm{cm}^{2}$ to $100 \mathrm{cal} / \mathrm{cm}^{2}$ dual layer switching jackets and pants $40 \mathrm{cal} / \mathrm{cm}^{2}$ to $100 \mathrm{cal} / \mathrm{cm}^{2}$ double layer switching hood. Protective goggles are still needed.

This classification establishes the importance of accurately identifying the type of exposure and the hazards that workers face in developing their work. Which was taken as a central element to consider in the process of identifying the types of protective clothing for work, the number of parts, components and materials like the color-coded by level of exposure.

\section{Methodology}

We develop an analysis of the worker's activities from the perspective of cognitive ergonomics in order to obtain structures that are part of work prevention strategies developed by experts-entry workers. Our objective is translated from the worker knowledge to the functions and operational objectives, integrating at the same time the use restrictions according to the contexts of action.

Our central objective of the transfer is to determine the functional and operational principles of the solution, taking into account the possible context of operation (which includes the largest number of variables of the future status of work) and the probable context of operation and functioning (and integrates the potential derives technical, operational and organizational, which operate the system or the developed solution.). By integrating these elements are also integrated the possible technical and organizational means to assist the user of the solution, in order to allow you to get the entirety of the benefits of the developed solution.

An ergonomic solution must meet two requirements, the first to effectively integrate the knowledge embedded in the state of activity being studied, second in its realization including the possibility of making this knowledge visible and time should be given the chance develop new knowledge.

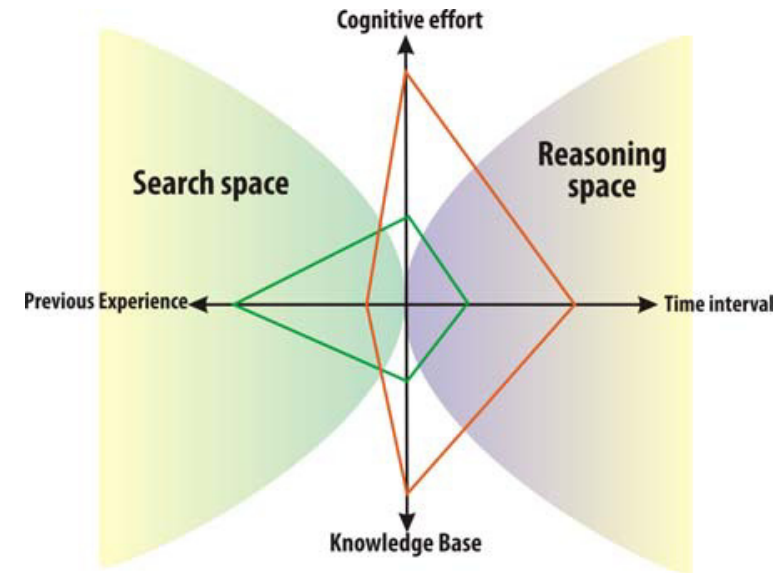

Figure 1. Analysis model used to identify the representations of the electrical workers, expressing his experience in the use of work clothes. 
According to principles of analysis previously established, we designed a study carried out following three steps:

2.1 Pre-evaluation. This phase aimed to specify the types of tasks and their characteristics, conducted the following activities:

a. Analysis of data on workers.

b. Objectives of the assignment. It seeks to establish what the goal to achieve in terms of timing, quality and efficiency.

c. Sequence of activities: it is to establish the use of work clothes (recruitment, travel, transportation of materials, operations performed by the employee)

d. Preliminary assessment of work clothes. Evaluation prior to use in terms of quality, materials, finishes, aesthetics, operation.

\subsection{Survey:}

It makes the application of a survey evaluating the ergonomic comfort perceived by the workers participating in the test. The survey was developed using a graphic medium in which they identify the following criteria: effectiveness, efficiency, satisfaction, flow, and expectation of Use.

2.3 Fieldwork: Information was collected using an array of analysis which records the perceptions of workers in the labor situation on the different items used, the purpose of this section is to associate the difficulties of using the allocation to activities are developing workers.

2.4 Characteristics of the activities reviewed.

The analyzed set of activities developed in public spaces, with specific activities in confined spaces (manholes interventions). To carry out the tasks required carrying workers' tools, media (Avantel, Cellular), measuring equipment and lighting. Additionally, some of them carrying harness for access to high points of intervention. Workers use different means of transport for journeys (truck, motorcycle, walking). Workers using the motorcycle as a means of transport must additionally comply with safety rules (helmet, vest and protection). Another aspect is the problem of access to service points, these vary depending on the sites of care (geographical location of neighborhoods, building types, connection types, age of facilities).

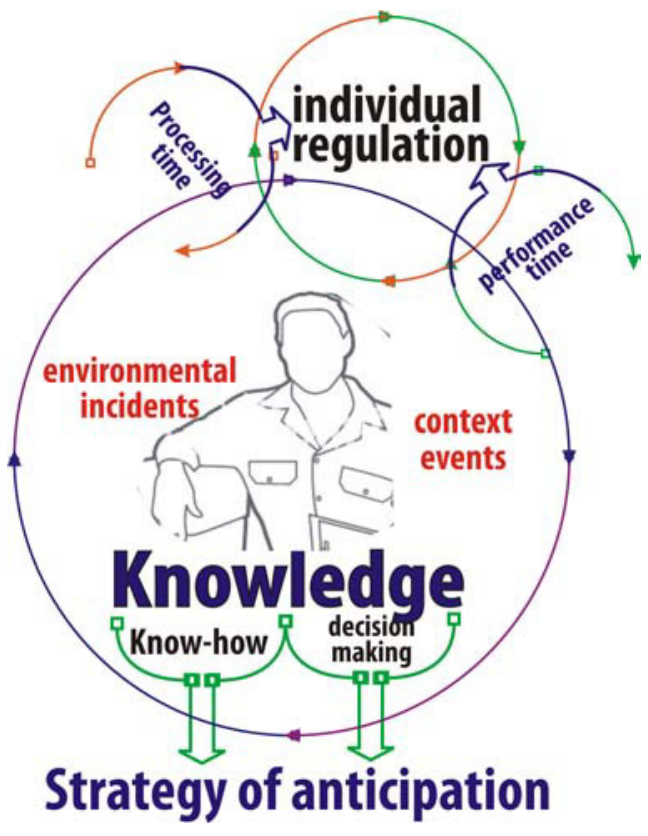

Figure 2. These are the concepts used in the analysis, recording and observation of the activities of the farm worker in direct assignments. Are used, as reference to identify which of them is predominant in each task analyzed.

\section{Results.}

Geographical areas of care users have a high influence on the duration of tasks, security restrictions and traffic. Some of the activities reviewed by the demands of intervention make endowments are exposed to wear and rapid aging or premature, this aspect is important to consider in the design of endowments.

Work activities involve developing civil works, demolition or modification of facilities, workers must make provision having alternating, since the physical demands and job type (finish building) damages the base capacity and affects institutional image. In these cases we must think of providing overalls or other specific provision for these tasks.

We found that the design of the envelope according to analysis of the activities carried out, it requires the use of work clothing and specifies differently, this means that activities in which it makes use of tools and data logging equipment must be attached to specific elements of their transportation to physical deployment activities should consider using an overall and for specific activities involving the use of 
motorcycles to develop an additional piece to protect the climate variation. Generally identifies activities that require the use of movement with spacious enclosures with ranges beyond the midline of the workers. This involves having a staff adapted to the dimensions of workers, but the crew used poses serious problems of length, asymmetries (different length shirts with left-right). Additionally, long shirts and tees are excessive and difficult to use, because it affects the mobility of upper and lower members.

Regarding the physical properties of the components of work clothing, we identified multiple problems assessed quality of material used in the first place and clothing and quality of inputs used in making the work clothing. This is seen in the rapid discoloration of the actual elements of the clothing work (after three washed process this pieces lost up to $50 \%$ of its original color).

Regarding work clothes can be indicated that workers perceive favorable change, however expressed fear of the confusion this may cause to users. On the other hand it was found that workers would prefer an endowment with corporate colors and logos of the company, it is suggested to have the logos of the companies providing services and the parent company. Additionally it is suggested to analyze the color composition of the envelope and that it must obey the most exposed areas, such as shirt collars and coats should better withstand exposure to environmental factors and the physical characteristics of workers (sweating). Then there is that the funding should be complemented with other elements such as transport of equipment bags (bills), also waterproof for workers and emergency reading. For the night shift should provide thermal shirts.

The study of work, then becomes the fundamental basis of control of the accident, as it seeks to develop tools to prevention of accidents. Always bearing in mind that work situations are characterized by a work environment characterized by instability and variability. Ergonomics addresses then the work in its dynamics and its complexity is not reduced to simple execution of operations, in which the individual is a subsidiary element of the system. The ergonomics through the study of the complexities of each activity, requires each of the components of working practices, this includes forecasting, anticipation and security.

These practices developed by the workers, differ in most cases the "standard practices" as defined in the manuals of procedure, in some cases openly violate safety rules and yet stabilizing the production systems and are source that allows productivity goals.

The integration of the informal activity practices, in the design of solutions allows us to consider the mediating character of "practice" and thus study their function as a tool in risk management. These practices are tools developed by workers in action, are designed to maintain control and security in the continuity of the process. Thus, these practices have an ecological character, in the sense that these are sensitive to the configuration of the situation evolving, this characteristic means that these practices are part of a history and a particular culture.

Thus a security measure constitutes a "data network" of different nature that not only guide action, but also will provide a platform for identification of possible scenarios. The study of the interactions among these elements enables design prevention strategies at work. More precisely this study try to understand the domains in which security measures should be developed. Besides the interactions that occur between these three elements can be presented as follows:

- The interaction technology / organization, privileging the study of the events. This aims to establish control measures and rules of procedure.

- In the interaction of worker organization, seeks to explore attitudes toward risk. It seeks to explain the reasons for the conduct of individuals. The aim is to understand the causes of adoption of certain behaviors to events.

- The worker interaction technology, interest focuses on understanding and identification of practices by individuals to risk management.

As a result of this analysis of design models of action, it is possible to identify that in the synchronous process safety management, which require electrical work activities, it becomes evident the need to integrate the individual's action in terms of knowledge and work practices, and consider the use of the experience, the latter acquire a core value in the structuring of activities and interventions that can maintain control and stability of a process. The contextual nature of the activity requires treatment to identify the elements developed by individuals for risk management, description of the context and knowledge that relate to actions in sync with the goals and objectives of the intervention. 


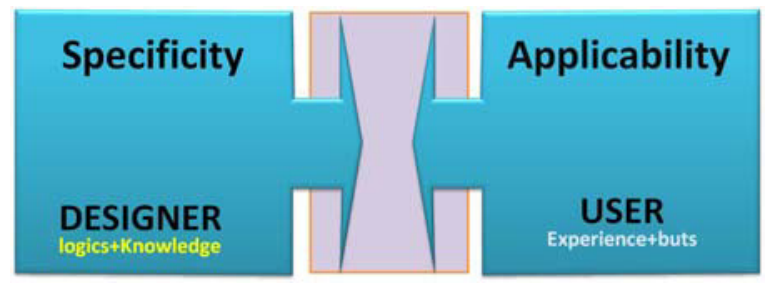

Figure 3. Development of design criteria must take into consideration the differences in logical elaboration of criteria made by the designer's work clothes, and those that are developed by the user, ie an employee who performs work according to the strategies us better suited to objectives and requirements of the assigned tasks.

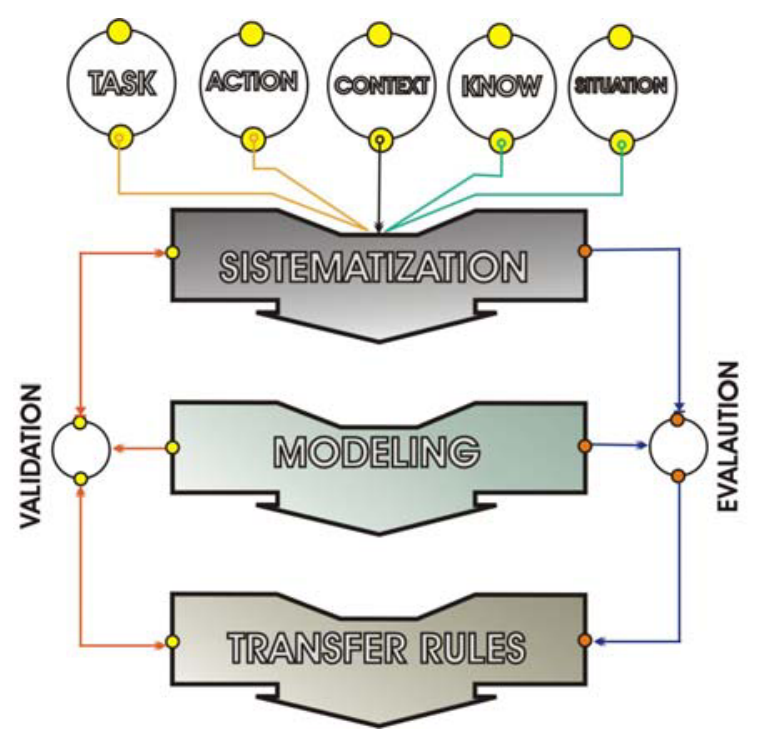

Figure 4. The model presents the concepts that must be integrated into the design process work clothes, these elements are obtained from a record-oriented ergonomic analysis of protection and prevention strategies that are specific to the activities developed by different teams workers.

It is important to remember that Basics design process is a process of knowledge transfer work activities to the technical specifications of the working clothes

\section{References}

Amalberti, R. (1996). La conduite de systèmes à risques. Paris, PUF.

Reason, J.(1990). L'erreur Humaine, Paris, PUF

Hubault, F. (2003). Quel type de management pour organiser, gérer cette prévention conjointe ?. papier présente au septième carrefour santé, sécurité au travail , U. Paris I.

Davezies, P. (1993). Eléments de Psychodynamique du travail, Education Permanente, 116, 3334.

Marine C. Cellier J.M. Vallax M.F. (1988). Dimensions de l'expertise dans une tâche de régulation de trafic: règles de traitement et profondeur du champs spatio-temporel Psychologie Française, 1988, 33, 3, 151-160.

Hoc J.M. (1989). La conduite d'un processus à long délais de réponse : une activité de diagnostic. Le Travail Humain, 52 , 4, 289-316.

Hoc, J.-M. and Rogalsky, J. (1992). Régulation des activités cognitives 